\title{
Helmintos gastrintestinais de capivaras (Hydrochoerus hydrochaeris) na sub-região de Paiaguás, Pantanal do Mato Grosso do Sul, Brasil
}

\author{
Gastrintestinal helminths of capybara (Hydrochoerus hydrochaeris) from \\ the Paiaguás subregion, in the floodplain of "Mato Grosso do Sul", Brazil
}

\author{
Marcos Roberto Bonuti ${ }^{1}$; Adjair Antonio do Nascimento ${ }^{2 *}$; \\ Elaine Bernardo Mapelli ${ }^{1}$; Isaú Gouveia Arantes ${ }^{2 *}$
}

\section{Resumo}

\begin{abstract}
Em trinta espécimes de capivaras (Hydrochoerus hydrochaeris hydrochaeris L., 1766) foram identificadas seis espécies de nematódeos, três de cestódeos e cinco de trematódeos. As espécies de maior prevalência foram: Protozoophaga obesa (96,66\%), Viannella hydrochoeri $(93,33 \%)$, Hydrochoerisnema anomalobursata (90,0\%), F.I. P. obesa (80,0\%), Taxorchis schistocotyle $(76,66 \%)$, Hippocrepis hippocrepis (70,0\%), Capillaria hydrochoeri (63,33\%), F.I. (Viannella + Hydrochoerisnema) (60,0\%), Strongyloides chapini (56,66\%), Monoecocestus macrobursatum (56,66\%), F.I. Monoecocestus (46,66\%), M. hydrochoeri (23,33\%) e M. hagmanni (23,33\%). Por outro lado, Trichostrongylus axei, Neocotyle neocotyle, Nudacotyle valdevaginatus e $N$. tertius ocorreram em níveis mais baixos. $\mathrm{O}$ total de helmintos identificados nas trinta capivaras foi de 236.097, sendo constatado $110(0,05 \%)$ exemplares nos estômagos, 49.240 (20,85\%) nos intestinos delgados e, 186.747 (79,10\%) nos intestinos grossos. As maiores variações de intensidade foram obtidas por P. obesa, F.I. P. obesa, F.I. (Viannella + Hydrochoerisnema); V. hydrochoeri, S. chapini e N. valdevaginatus com média de 3.900,0 (04-20885), 2.958,1 (40-18130), 689,7 (05-7515), 601,5 (10-4585), 765,3 (05-4055) e 1.270,0 (0-1270) helmintos por animal, respectivamente. $V$. hydrochoeri e $S$. chapini são as espécies mais patogênicas, com parasitoses clínicas e subclínicas em capivaras.

Palavras chave: Helmintos; Capivaras; Hydrochoerus hydrochaeris hydrochaeris; Prevalência.
\end{abstract}

\begin{abstract}
Six nematode, three cestode and five trematode species were identified in 30 capybara specimens (Hydrochoerus hydrochaeris hydrochaeris L., 1766). The species of greater prevalence were: Protozoophaga obesa (96.66\%), Viannella hydrochoeri (93.33\%), Hydrochoerisnema anomalobursata (90.0\%), F.I. P. obesa (80.0\%), Taxorchis schistocotyle (76.66\%), Hippocrepis hippocrepis (70.0\%), Capillaria hydrochoeri (63.33\%), F.I. (Viannella + Hydrochoerisnema) (60.0\%), Strongyloides chapini (56.66\%), Monoecocestus macrobursatum (56.66\%), F.I. Monoecocestus (46.66\%), M. hydrochoeri (23.33\%) and M. hagmani (23.33\%). On the other hand, Trichostrongylus axei, Neocotyle neocotyle, Nudacotyle valdevaginatus and $N$. tertius occurred at lower levels. The total of helminths identified in the 30 animals was of 236,097 being verified $110(0.05 \%)$ specimens in the stomach, 49,240 $(20.85 \%)$ in the small intestines, 186,747 (79.10\%) in the large intestines. The higher intensity variation were obtained by P. obesa, F.I. P. obesa, F.I. (Viannella + Hydrochoerisnema); V. hydrochoeri, S. chapini e $N$. valdevaginatus, with a mean of 3,900.0 (04-20,885), 2,958.1 (40-18,130), $689.7(05-7,515), 601.5$ $(10-4,585), 765.3(05-4,055)$ and 1,270.0 (0-1,270) worms per animal, respectively. $V$. hydrochoeri and $S$. chapini are the most pathogenic species, with clinical and subclinical parasitosis in capybara.
\end{abstract}

Key words: Helminths; Capybara; Hydrochoerus hydrochaeris hydrochaeris; Prevalence

\footnotetext{
1 Alunos do Curso de Pós-Graduação em Medicina Veterinária - Medicina Veterinária Preventiva, Nível Doutorado - FCAV UNESP, Jaboticabal, Fone: (17)442-1093. E-mail: bonuti@bol.com.br

2 Professor Assistente, Doutor, Faculdade de Ciências Agrárias e Veterinárias UNESP, CEP: 14870-000 - Campus de Jaboticabal.

* Autores para correspondência.
} 


\section{Introdução}

A capivara é um roedor de hábitos semi-aquáticos que encontra-se em fase de domesticação em várias regiões do país e constitui um grupo de animais herbívoros pertencentes à Ordem Rodentia e à família Hydrochoeridae, sendo encontrada no Brasil uma única subespécie: Hydrochoerus hydrochaeris hydrochaeris L., 1766. As maiores concentrações desses animais são encontradas nas zonas inundáveis da Colômbia, da Venezuela, do Paraguai e do Brasil (área do Pantanal).

Este animal é parasitado por mais de duas dezenas de helmintos, que determinam lesões nos intestinos delgado e grosso e nos vasos sangüíneos dos rins, pulmões e coração, sendo descritos por Travassos (1916); Travassos (1918); Travassos (1923); Rego (1961); Travassos; Freitas e Kohn (1969); Arantes e Artigas (1980); Arantes e Artigas (1983).

O parasitismo em capivaras, provenientes da região do Pantanal do Mato Grosso do Sul, foi observado por Arantes (1983), Arantes et al.(1985), Nascimento et al.(1991), Nascimento et al.(1992), Costa e Catto (1994) e Nascimento et al.(1996); na Argentina, foi verificado por Boero e Boehringer (1967), Lombardero e Moriena (1973) e Sutton et al. (1997) e na Bolívia por Casas et al. (1995).

Os estudos parasitológicos de capivaras são escassos e quase sempre voltados à sistemática de helmintos. Portanto objetiva-se, nessa oportunidade, catalogar as espécies de helmintos de capivara, avaliar em condições naturais a carga parasitária, a intensidade, a intensidade média, a abundância e a prevalência das diversas espécies de parasitos, e dessa maneira espera-se obter subsídios, para que se possa melhor entender as infecções helmínticas desses animais, que se encontram em fase de domesticação em diversas regiões fisiográficas do país.

\section{Material e métodos}

Foram utilizadas 30 capivaras naturalmente infectadas, sendo 03 jovens (menos de 06 meses de idade) e 27 adultas, de ambos os sexos, capturadas entre 1987 e 1996, nos municípios de Pedro Gomes (12 animais), Corumbá (14 animais) e Coxim (04 animais), cujos sacrifícios foram autorizados anualmente pelo Instituto Brasileiro do Meio Ambiente e dos Recursos Naturais Renováveis (IBAMA).

Após a captura os animais foram sacrificados por sangria direta dos grandes vasos, semelhante ao abate de suínos. Posteriormente o trato gastrintestinal foi separado em suas divisões anatômicas (estômago, intestinos delgado e grosso). A seguir, cada segmento foi aberto em bandeja de metal e suas mucosas lavadas com água corrente. O material obtido foi tamisado, utilizando-se tamises de $100 \mathrm{~mm}$ de abertura. O conteúdo assim obtido foi fixado com líquido de Railliet e Henry, de acordo com a técnica preconizada por Travassos (1950), e acondicionados em garrafas identificadas.

Os helmintos foram colhidos de uma amostra de $20 \%$, do conteúdo total de cada segmento gastrintestinal analisado com o auxílio de microscópio estereoscópico, separados por sexo e mantidos em líquido fixador de Railliet e Henry, para serem posteriormente contados e identificados. Os valores de intensidade; intensidade média; abundância e prevalência, foram calculados de acordo com Margolis et al. (1982).

\section{Resultados e discussão}

Identificaram-se nos trinta animais examinados quatorze espécies de helmintos, sendo cinco espécies de trematódeos: Nudacotyle valdevaginatus; Nudacotyle tertius; Neocotyle neocotyle; Hippocrepis hippocrepis e Taxorchis schistocotyle; seis espécies de nematódeos: Trichostrongylus axei; Viannella hydrochoeri; Hydrochoerisnema anomalobursata; Strongyloides chapini; Capillaria hydrochoeri; Protozoophaga obesa e três espécies de cestódeos: Monoecocestus hagmanni; Monoecocestus macrobursatum e Monoecocestus hydrochoeri.

No que tange à estrutura da fauna helmíntica, esses resultados assemelham-se aos de Costa e Catto 
(1994), também verificados no Pantanal Matogrossense (Nhecolândia), que observaram 12 espécies de helmintos e diferem dos estudos realizados em capivaras na Argentina, Venezuela e Bolívia (BOERO; BOEHRINGER, 1967; LOMBARDERO; MORIENA,1973; TARBES ,1980; CASAS et al.,1995; SUTTON et al., 1997), que constataram o parasitismo por 26 espécies de helmintos.
Todas as 30 capivaras examinadas apresentaram infecções helmínticas. O número total de helmintos nos tratos gastrintestinais foi de 236.097, tendo sido observado no mínimo 01 e no máximo 113.069 espécimes. Nos intestinos delgado e grosso foi encontrada a maior quantidade de helmintos (Tabela 1).

Tabela 1 - Indicadores das infecções naturais de helmintos observados nos segmentos gastrintestinais de 30 capivaras, procedentes da região do Pantanal, Mato Grosso do Sul.

\begin{tabular}{|c|c|c|c|c|c|c|c|}
\hline \multirow{2}{*}{$\begin{array}{l}\text { Espécies de vermes } \\
\text { Trichostrongylidae }\end{array}$} & \multirow[t]{2}{*}{ Habitat } & \multicolumn{6}{|c|}{ Indicadores das infecções } \\
\hline & & Variação intensidade & $\begin{array}{l}\text { Intensidade } \\
\text { média }\end{array}$ & Abundância & Prevalência & Total de helmintos & $(\%)$ \\
\hline Trichostrongylus axei & Estômago & $5-45$ & $27,5(4)$ & 3,66 & 13,33 & 110 & 0,05 \\
\hline \multicolumn{8}{|l|}{ Viannaiidae } \\
\hline Viannella hydrochoeri & I. delgado & $10-4.587$ & $601,5(28)$ & 561,36 & 93,33 & 16.841 & 7,13 \\
\hline $\begin{array}{l}\text { Hydrochoerisnema } \\
\text { anomalobursata }\end{array}$ & & $05-638$ & $127,4(27)$ & 114,63 & 90,00 & 3.439 & 1,46 \\
\hline $\begin{array}{l}\text { Forma imatura (Viannella }+ \\
\text { Hydrochoerisnema) }\end{array}$ & & $05-7.515$ & $689,7(18)$ & 413,83 & 60,00 & 12.415 & 5,26 \\
\hline \multicolumn{8}{|l|}{ Strongyloididae } \\
\hline Strongyloides chapini & & $05-4.055$ & $765,3(17)$ & 433,66 & 56,66 & 13.010 & 5,51 \\
\hline \multicolumn{8}{|l|}{ Trichuridae } \\
\hline \multicolumn{8}{|l|}{ Anoplocephalidae } \\
\hline Monoecocestus hagmanni & & $05-55$ & $21,4(7)$ & 5,0 & 23,33 & 150 & 0,06 \\
\hline Monoecocestus macrobursatum & & $05-195$ & $40,9(17)$ & 23,16 & 56,66 & 695 & 0,29 \\
\hline Monoecocestus hydrochoeri & & $05-130$ & $50,7(7)$ & 11,83 & 23,33 & 355 & 0,15 \\
\hline Forma imatura de Monoecocestus & & $05-90$ & $23,6(14)$ & 11,00 & 46,66 & 330 & 0,14 \\
\hline \multicolumn{8}{|l|}{ Nudacotylidae } \\
\hline Nudacotyle valdevaginatus & & $0-1.270$ & $1.270,0(01)$ & 42,33 & 3,33 & 1.270 & 0,54 \\
\hline Nudacotyle tertius & & $0-95$ & $95,0(01)$ & 3,16 & 3,33 & 95 & 0,04 \\
\hline Neocotyle neocotyle & & $0-01$ & $1,0(01)$ & 0,033 & 3,33 & 01 & 0,0004 \\
\hline \multicolumn{8}{|l|}{ Oxyuridae } \\
\hline Protozoophaga obesa & I. grosso & 04-20.885 & $3.900,0(29)$ & 3,77 & 96,66 & 113.069 & 47,89 \\
\hline $\begin{array}{l}\text { Forma imatura de Protozoophaga } \\
\text { obesa }\end{array}$ & & $40-18.130$ & $2.958,1(24)$ & $2.366,5$ & 80,00 & 70.995 & 30,07 \\
\hline \multicolumn{8}{|l|}{ Nudacotylidae } \\
\hline Hippocrepis hippocrepis & & $05-380$ & $107,8(21)$ & 75,50 & 70,00 & 2.265 & 0,96 \\
\hline \multicolumn{8}{|l|}{ Cladorchiidae } \\
\hline Tachorxis schistocotyle & & $02-65$ & $18,2(23)$ & 13,93 & 76,66 & 418 & 0,18 \\
\hline TOTAL & & & & & & 236.097 & \\
\hline
\end{tabular}

( ) = número de animais parasitados

Do total de helmintos colhidos, P. obesa representou 77,96\% (184.064 exemplares), as demais espécimes representaram $22,06 \%(55.035)$ da carga parasitária total, assemelhando-se em parte aos apresentados por Costa e Catto (1994). Essa espécie constitui o mais comum nematódeo parasito de capivaras, pois apresentou os valores mais significativos dos indicadores das infecções: intensidade (04-20.885), intensidade média (3.900), abundância $(3.768,9)$ e prevalência $(96,66 \%)$.

T. axei parasita bovinos, bubalinos, caprinos, ovinos, eqüinos, asininos, suínos (COSTA et al., 1986), cervídeos e taiassuídeos (NASCIMENTO et al. 1991; 1996). Em capivaras, no Mato Grosso do Sul, no- 
tou-se prevalência de 69,2\% e intensidade média de 35 helmintos (ARANTES,1983; ARANTES et $a l ., 1985)$ e em outro estudo a prevalência foi de 60,9\% e a intensidade média de 14 vermes (COSTA; CATTO,1994). Constata-se, portanto divergências entre os dados obtidos e os verificados por aqueles autores, embora os valores de intensidade média $(27,5)$ assemelham-se aos apresentados por ARANTES et al. (1985). Com relação à origem do parasitismo do T. axei na capivara, levando-se em consideração unicamente o espectro de hospedeiros, discordamos da hipótese de Costa e Catto, na qual as infecções são oriundas de eqüinos e bovinos.

A prevalência de $V$. hydrochoeri foi de $93,33 \% \mathrm{e}$ a intensidade média de 601,50; semelhantes aos encontrados por Costa e Catto(1994) e Arantes (1983), divergindo dos relatados por Casas et al. (1995) e Tarbes (1980) que assinalam baixos valores para estes indicadores.

O parasitismo de capivaras por $S$. chapini foi notificado por Arantes et al. (1985) em animais procedentes dos Estados de São Paulo e do Mato Grosso do Sul, sendo que no presente estudo observaram-se prevalência de $56,66 \%$ e intensidade média de 765,3 nematódeos (Tabela 1), semelhantes aos dados apresentados por Costa e Catto(1994).

Observações feitas no Brasil, por Nascimento et al. (1991) e Costa e Catto(1994) sobre infecções de C. hydrochoeri em capivara, caracterizam-se por apresentarem baixos valores de intensidade, intensidade média e de abundância. Por outro lado, dados pertinentes a prevalência obtidos por Costa e Catto e nossos atuais relacionam índices mais expressivos de $86,9 \%$ e de $63,33 \%$ e, portanto próximos.

Taxorxis schistocotyle tem por habitat a mucosa ceco-cólica onde se fixam. A presença deste helminto nas Américas fora assinalada, no Brasil por Travassos (1922), Arantes et al. (1985), Nascimento et al. (1991) e Costa e Catto (1994); na Argentina por Lombardero e Moriena (1973); na Venezuela por Tarbes (1980) e na Bolívia por Casas et al. (1995). Com relação aos indicadores de infecções, prevalência e intensidade média observados por Costa e Catto(1994) e Casas et al. (1995) expressam valores de 56,5\%, 43 e 12\%, 10, respectivamente. Neste particular, os resultados atuais $(76,6 \%$ e 18,2$)$ diferem ao menos em parte daqueles obtidos pelos autores.

Hippocrepis hippocrepis, que são comumente encontrados aderidos a mucosa do colo, foi notificado no Brasil por Travassos (1922), Nascimento et al. (1991), Hoffmann et al. (1986), Costa e Catto(1994); na Bolívia por Casas et al. (1995); na Argentina por Lombardero e Moriena (1973) e Sutton et al. (1997) e na Venezuela por Rodriguez; Brito; Perea (1975) e Tarbes (1980). Costa e Catto(1994) registraram prevalência de $34,8 \%$ e intensidade média de 3832 vermes e Casas et al. (1995) prevalência de $20 \%$ e intensidade média 10. Em nossas observações verificamos intensidade média de 107,8 e 70\% de prevalência (Tabela 1). Esses resultados divergem dos observados por aqueles autores.

Nudacotyle valdevaginatus e $N$. tertius foram descritos no Brasil por Travassos (1922; 1939); respectivamente. Costa e Catto (1994) verificaram prevalência de $8,7 \%$ e intensidade média de 133 para $N$. tertius no pantanal de Nhecolândia, MS, resultados estes semelhantes ao presente estudo no pantanal de Paiaguás.

Neocotyle neocotyle, com presença nas Américas assinalada por Travassos (1922) no Brasil e por Sutton et al. (1997) na Argentina, apresentou no presente estudo prevalência de $3,33 \%$ e intensidade média de 1270, indicadores de infecção não registrados por outros autores.

O gênero Monoecocestus foi revisado por Rego (1961) e, segundo este autor, as capivaras no Brasil são parasitadas por M. hagmanni; $M$. hydrochoeri $\mathrm{e}$ M. macrobursatum, espécies também observadas na Bolívia por Casas et al. (1995) com prevalências de 12,12 e $34 \%$, respectivamente. Por outro lado, o parasitismo das capivaras da Argentina são por $M$. hydrochoeri, Boero e Boehringer (1967) e da Venezuela por M. hagmanni, Tarbes (1980). As espécies registradas, com suas intensidades médias e 
prevalências, foram: M. hagmanni (21,4 e 23,33\%); M. macrobursatum (40,9 e 56,66\%) e M. hydrochoeri (50,7 e 23,33\%), diferentes aos apresentados por Costa e Catto(1994) para M. hagmanni (75e 80\%) e $M$. hydrochoeri (54,5 e 62\%), bem como, aos apresentados por Casas et al. (1995) quanto as prevalências.

Analisando os resultados obtidos, podemos sugerir que as principais espécies de helmintos que possuem grande potencial patogênico e podem ser consideradas como sérios riscos à manutenção da saúde dos animais jovens nascidos no cativeiro, são: Strongyloides chapini, Viannella hydrochoeri e Trichostrongylus axei.

A expectativa criada a respeito da intensidade e da abundância das infecções helmínticas naturais de capivaras que habitavam a área em estudo do Pantanal do Mato Grosso do Sul, foi confirmada possivelmente em decorrência da grande concentração da espécie ao redor das coleções de água persistentes nos campos (baías e vazantes), por mais de 180 dias anteriores ao período de captura.

Com relação a importância das infecções helmínticas da capivara no cativeiro, pode-se concluir que condições ambientais adequadas favorecem o desenvolvimento e sobrevivência das formas pré-parasíticas dos helmintos no ambiente, implicando em maiores possibilidades de infecção dos hospedeiros em suas faixas etárias de maior susceptibilidade. V. hydrochoeri e S. chapini são as espécies mais patogênicas.

\section{Referências}

ARANTES, I. G. Considerações sobre Trichostrongyloidea Cram, 1927, parasitas de capivaras (Hydrochoerus hydrochaeris hydrochaeris L. 1766) provenientes do estado do Mato Grosso do Sul (Municípios de Angélica, Aquidauana e Rio Verde). 1983. Tese (Livre Docência) - Faculdade de Ciências Agrárias e Veterinárias, Universidade Estadual Paulista, Jaboticabal.

ARANTES, I. G.; ARTIGAS, P. T. Hydrochoerisnema anomalobursata parasita de Hydrochoerus hydrochaeris (Rodentia: Hydrochoeridae). In: CONGRESSO BRA-
SILEIRO DE PARASITOLOGIA, 1980, Rio de Janeiro. Anais... Rio de Janeiro: UFRRJ, 1980. p.98

ARANTES, I. G.; ARTIGAS, P. T. Hydrochoerisnema anomalobursata Arantes \& Artigas, 1980 (Nematoda; Trichostrongyloidea) parasito de capivara (Hydrochoerus hydrochaeris hydrochaeris, L. 1766). Estabelecimento de nova sub família: Hydrochoerisnematinae. Arquivo Instituto Biológico, São Paulo, v.50, n.1/4, p.39-49, 1983.

ARANTES, I. G. et al. Trichostrongyloidea Cram, 1927, Parasitas de Capivaras (Hydrochoerus hydrochaeris hydrochaeris, L. 1766) provenientes do Estado de Mato Grosso do Sul, Brasil. In: ENCONTRO DE PESQUISAS VETERINÁRIAS, 10., 1985, Jaboticabal. Anais... Jaboticabal : UNESP, 1985, p.63

BOERO, J. J.; BOEHRINGER, I. K. El parasitismo de nuestra fauna autóctona. II. Los parasitos del carpincho (Hydrochoerus hy drochaeris) y del Quijá (Myocastor coypus). Revista de la Faculta de Ciencias Veterinarias La Plata, La Plata, v.9, n.21, p.161-171, 1967.

CASAS, M. C. et al. Intestinal Helminths of Capybara (Hydrochaeris hydrochaeris) from Bolivia. Journal of the Helminthological Society of Washington, Lawrence, v.62, n.1, p.87-88, 1995.

COSTA, H. M. A. et al. Distribuição dos helmintos parasitas de animais domésticos no Brasil. Arquivo Brasileiro Medicina Veterinária Zootecnia, Belo Horizonte, v.38, p.465-579, 1986.

COSTA, C. A. F.; CATTO, J. B. Helmintos parasitos de capivaras (Hydrochoerus hydrochaeris) na sub-região da Nhecolândia, Pantanal Sul Matogrossense. Revista Brasileira Biologia, Rio de Janeiro, v.54, n.1, p.39-48, 1994

HOFFMANN, R. P. et al. Ocorrências de helmintos em capivaras (Hydrochoerus hydrochoerus). Arquivo da Faculdade de Veterinária da UFRGS, Porto Alegre, v.14, p.71-75, 1986.

LOMBARDERO, O. J.; MORIENA, R. A. Nuevos helmintos del carpincho (Hydrochoerus hydrochaeris) para la Argentina. Revista de Medicina Veterinaria, São Paulo, v.53, n.3, p.265-269, 1973.

MARGOLIS, L. et al. The use of ecological terms in parasitology (Report of na Ad Hoc Committee of the American Society of Parasitologists). Journal of Parasitology, Lawrence, v.68, n.1, p.131-133, 1982.

NASCIMENTO, A. A. et al. Helmintos Parasitos de Hydrochoerus hydrochaeris hydrochaeris (Linnaeus, 1766) no Mato Grosso do Sul, Brasil. Revista Brasileira Parasitologia Veterinária, São Paulo, v.1, n.0, p.95, 1991. 
NASCIMENTO, A.A. et al. Dipetalonema (Alafilaria) hydrochoerus, Yates e Jorgenson, 1983, parasito de capivara (Hydrochoerus hydrochaeris hydrochaeris, L. 1766) no Estado do Mato Grosso do Sul, Brasil. In: ENCONTRO DE PESQUISAS VETERINÁRIAS, 14., 1992, Jaboticabal. Anais... Jaboticabal : UNESP, 1992. p.189

NASCIMENTO, A. A. et al. Nematódeos Filarioidea parasitos de capivaras (Hydrochoerus h. hidrochaeris) procedentes do Mato Grosso do Sul. In: CONGRESSO BRASILEIRO DE MEDICINA VETERINÁRIA, 24., 1996, Goiania. Anais... Goiânia: UNESP, 1996. p.234

REGO, A.A. Revisão do gênero Monoecocestus Beddard, 1914 (Cestoda: Anoplocephalidae). Memoria do Instituto Oswaldo Cruz, Rio de Janeiro, v.59, n.3, p.325-354, 1961.

RODRIGUEZ, H. C.; BRITO, E. C. ; PEREA, F. L. Aspectos fisiológicos y sanitários del chigüire. Cespedesia, Bogotá, v.4, p.15-44, 1975.

SUTTON, C. A. et al. The Notocotyloidea digeneans from Hydrochaeris hydrochaeris Linne (Rodentia) in Argentina. Gayana Zoologia, Conscepcion, v.61, n.1, p.23-31, 1997.

TARBES, H. M. Sobre uma coleção de helmintos de chiguire (Hydrochoerus hydrochaeris) de Venezuela. Revista Facultad Ciencias Veterinaris U. C. V. Maracay, v.28, n.118, p.19-47, 1980.
TRAVASSOS, L. Informações sobre a fauna helmintológica sul-fluminense. II. Brasil Médico, Brasília, v.30, n.40, p.312-314, 1916.

Trichostrongylidae brasileiros. Revista Sociedade Brasileira Ciência, São Paulo, v.3, p.191, 1918.

Informações sobre a fauna helmintológica de Mato Grosso, Trematódeos. Folha Médica, São Paulo, v.3, n.24, p.187-190, 1922.

Informações sobre a fauna helmintológica do Mato Grosso. Oxyuroidea-oxyuridae. Folha-Médica, São Paulo, v.4, n.5, p.35-38, 1923.

Contribuições ao conhecimento de alguns trematódeos de Mato Grosso. Nudacotylinae Baker, 1916. Boletim Biologia, v.4, n.2, p.160-167, 1939.

. Introdução ao estudo da Helmintologia. Revista Brasileira Biologia, Rio de Janeiro, v.10, p.169, 1950.

TRAVASSOS, L.; FREITAS, J. F. T.; KOHN, A. Trematodeos do Brasil. Memorias do Instituto Oswaldo Cruz, Rio de Janeiro, v.67, p.1-886, 1969. 